\title{
SOMATOSTATINOMA DUODENAL
}

\section{DUODENAL SOMATOSTATINOMA}

\author{
Dr. Ferreira Bogado, Miguel'; Dra. Mosqueira Lara, María Nilda
}

\section{RESUMEN}

Alrededor del 70\%de los tumores endocrinos bien diferenciados asientan en el tracto gastrointestinal. Los tumores duodenales bien diferenciados constituyen solo el 2,6 \% de todos los tumores neuroendocrinos (NET).El somatostatinoma es un tumor raro que se localiza en páncreas o duodeno con una incidencia de 1:40 millones.

La neurofibromatosis tipo Ies una enfermedad autosómica dominante, la mutación en el gen supresor de tumores NF1 favorece la aparición de neoplasias en estos pacientes.

Palabras clave: tumor neuroendocrino, somatostatinoma.

\section{SUMMARY}

About $70 \%$ of well-differentiated endocrine tumors arise from the gastrointestinal tract. Duodenal well-differentiated tumors account for only $2.6 \%$ of all neuroendocrine tumors.Somatostatinomas are rare neuroendocrine tumors (NETs) with an incidence of 1 in 40 million. These unusual tumorsarise predominantly in the pancreas and peripancreaticduodenum.

Neurofibromatosis type I is an autosomal dominant disease, the mutation in the tumor suppressor gene NF1 favors the appearance of neoplasms in these patients.

Keywords: neuroendocrine tumor (NET), somatostatinoma

\section{INTRODUCCIÓN}

Los tumores neuroendocrinos (NET) derivan de las células enterocromafines del tracto gastrointestinal y fueron descritos por Lubarsch en $1.888^{1}$. Desde 1.974 , los tumores neuroendocrinos (NETs) se han clasificado de acuerdo con las principales hormonas secretadas, tales como insulinoma, gastrinoma, VIPoma, glucagonoma y somatostatinoma.

Desde los dos primeros casos de somatostatinoma informados en 1.977, menos de 200 casos de somatostatinoma han sido reportados ${ }^{2}$. El somatostatinoma es un tumor raro que se localiza en páncreas o duodeno con una incidencia de 1:40 millones, cuya presentación clínica es variable ${ }^{3}$. En los pacientes con neurofibromatosis tipo I la incidencia de este tipo de tumor es de alrededor al $1 \%$ y suelen asociarse con adenocarcinomas, tumores de GIST y feocromocitoma. Aquellos de localización duodenal presentan con menor frecuencia el síndrome del somatostatinoma caracterizado por diabetes mellitus, colelitiasis y esteatorrea convirtiendo en más difícil si cabe su diagnóstico preoperatorio ${ }^{5}$. Las manifestaciones clínicas más frecuentes en los no-funcionantes son: la ictericia (65\%), dolor abdominal inespecífico (31\%) junto con hemorragia digestiva baja, anemia ferropénica, oclusión intestinal, colangitis y pancreatitis ${ }^{5}$. En so- matostatinomas duodenales, el síndrome del somatostatinoma puede ocurrir sólo si el tumor es mayor de $4 \mathrm{~cm}^{6}$. Debido al potencial maligno de estos tumores el gold standard del tratamiento es la cirugía, considerándose la resección local o endoscópica de las lesiones menores de $1 \mathrm{~cm}^{7}$. El riesgo de metástasis aumenta significativamente en tumores de $2 \mathrm{~cm}$. Para tumores más grandes $(>2 \mathrm{~cm})$, la cirugía de Whipple con linfadenectomía regional debe ser considerada ${ }^{7}$.El tratamiento adyuvante con análogos de la somatostatina ha demostrado un aumento en la supervivencia.

\section{CASO CLÍNICO}

Paciente de sexo masculino de 47 años de edad, portador de Enfermedad de Von Recklinghausen, sin tratamiento. Refiere cuadro de 1 mes de evolución de vómitos en varias oportunidades, de contenido alimentario, precedido de náuseas. Se agrega al cuadro pérdida de peso de $15 \mathrm{~kg}$, coloración amarillenta de piel y mucosas, astenia y anorexia.

Examen físico: Piel y mucosas: neurofibromas y manchas café con leche en miembros superiores e inferiores, tórax y abdomen (Figura 1). Resto sin datos de valor.

Estudios laboratoriales: Hb. 12,7g/dl Hto. 37,9\% GB: 12.300 N 80\% B.T: 2,90 B.D: 1,3 F.A: 570 G.O.T: 57 G.P.T: 90 Amilasa: 1065 Lipasa: 2054 CEA 125 7,39 U/ml CA19-9 25,2 U/m

Ecografía abdominal: vías biliares intrahepáticas dilatadas,

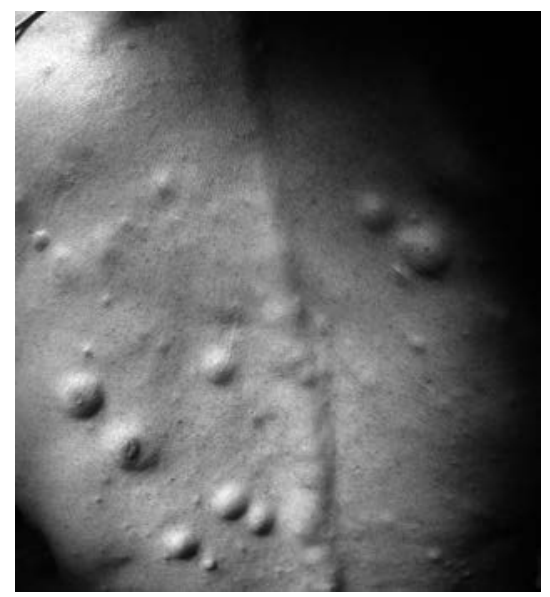

Figura 1. Neurofibromas y manchas café con leche 


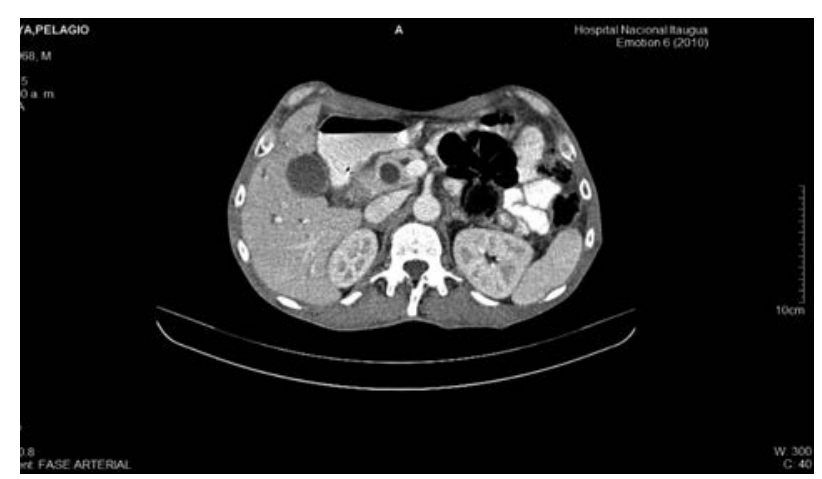

Figura 2. La tomografía computarizada de abdomen muestra una masa bien definida en la segunda porción del duodeno.

colédoco dilatado, mide $16 \mathrm{~mm}$ de diámetro, vesícula biliar: aumentada de volumen, pared no engrosada, contenido barro biliar. Páncreas no visualizado por interposición de gases. Se constata una imagen heterogénea en flanco derecho. En TAC de abdomen se observa dilatación del conducto biliar y pancreático y un tumor de 3,5 cm en región periampular (Figura 2). Se realiza EDA que informa en la 2da. porción del duodeno lesión de aspecto adenomatoso que ocupa casi toda la circunferencia. Se recibe informe de biopsias: en la lámina propia un adenocarcinoma moderadamente diferenciado. Se indica cirugía, realizándose una duodenopancreatectomía cefálica (Figura 3), la anatomía patológica informa tumor neuroendocrino bien diferenciado de duodeno, que mide $6,5 \mathrm{~cm}$ e infiltra toda la pared muscular del duodeno. Muestra cuerpos de psammoma intraluminales. El tumor se ajusta a la clasificación de Somatostatinoma duodenal o periampular T3 N1 Mx Estadio IIIB.

\section{DISCUSIÓN}

La somatostatina es un péptido cíclico de 14 aminoácidos que es secretado por el hipotálamo, el cerebro, la médula espinal, el nervio vago y células D en los islotes de Langerhans del páncreas, estómago, el duodeno y el intestino delgado. La somatostatina inhibe numerosas hormonas secretoras endocrinas y exocrinas, incluyendo insulina, colecistoquinina, enzimas pancreáticas y gastrina ${ }^{6}$.

Clínicamente, en comparación con los somatostatinomas pancreáticos, los somatostatinomas duodenales se asocian más a menudo con síntomas inespecíficos y neurofibromatosis, pero menos frecuentemente con el síndrome del somatostatinoma o metástasis. El síndrome de somatostatina se produce sólo con somatostatinomas mayores que $4 \mathrm{~cm} .{ }^{6}$ En este caso, el tumor se

\section{BIBLIOGRAFÍA}

1. Lubarsch O. Uber den pimaerenkrebs des ileum nebst Bemerkungen über das gleichzeitige Vorkommen von krebs und tuberculos. Virchows Arch 1888;111:280-317.

2. Larsson LI, Hirsch MA, Holst JJ, Ingemansson S, Kuhl C, Jensen SL et al. Pancreatic somatostatinoma: clinical features and physiological implications. Lancet1977;1:666-8.

3. Kimura R, Hayashi Y, Takeuchi T, Shimizu M, Hiratsuka M, Yoshida $\mathrm{M}$, et al. Large duodenal somatostatinoma in the third portion associated with severe glucoseintolerance. Intern Med 2004;43:704-07.

4. Ganda OP, Weir GC, Soeldner JS, Legg MA, Chick WL, Patel YC et al. 'Somatostatinoma': a somatostatin containing tumor of the endocrine pancreas. N Engl J Med 1977; 296:963-7.

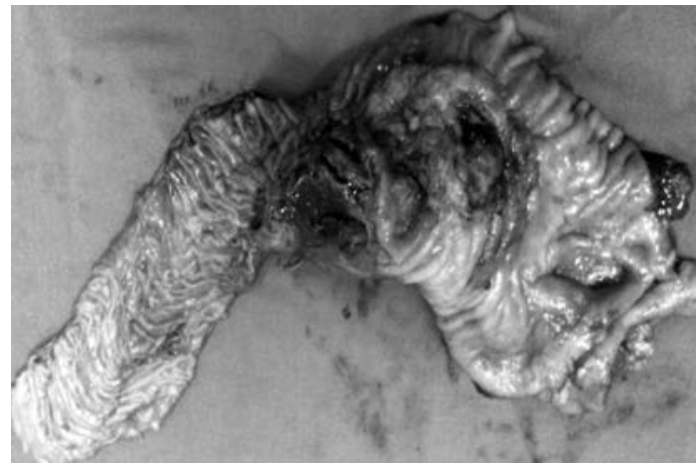

Figura 3. La pieza operatoria muestra una masa ovalada abultada en la luz duodenal.

originó en el duodeno, medía $6,5 \mathrm{~cm}$ y el paciente presentó vómitos incoercibles, pérdida de peso, ictericia, astenia y anorexia, además era conocido portador de Neurofibromatosis tipo I.

En pacientes con Neurofibromatosis tipo I la incidencia de este tipo de tumores es del $1 \%$.

La característica histológica más característica de los somatostatinomas es el cuerpo de psammoma. Estos cuerpos esféricos han sido reportados en $68 \%$ de los casos de somatostatinoma duodenal ${ }^{9}$.

Según los hallazgos patológicos nuestro tumor fue diagnosticado como un somatostatinoma duodenal.

Teniendo en cuenta su potencial maligno, la resección quirúrgica es el tratamiento recomendado en este caso. Generalmente, para tumores de $1 \mathrm{~cm}$ o menos, la escisión endoscópica es adecuada debido a la baja incidencia de metástasis. ${ }^{7}$ Para las lesiones entre 1 y $2 \mathrm{~cm}$, la escisión transduodenal debe ser aplicada.

Es probable que los tumores mayores de $1 \mathrm{~cm}$ invadan a la capa profunda más allá de la submucosa y la resección endoscópica no puede siempre extraer el tumor por completo ${ }^{7}$.

La naturaleza maligna de los somatostatinomas duodenales y el riesgo metástasis aumentan significativamente en tumores mayores a $2 \mathrm{~cm}$.

En el paciente se realizó la cirugía de Whipple, teniendo en cuenta que el tamaño del tumor era mayor a $2 \mathrm{~cm}$. Durante el post operatorio presentó una fístula quilosa que fue tratada con dieta hipograsa, hiperproteica, NPT y análogos de la somatostatina. Fue dado de alta al 30mo. día post operatorio.

La supervivencia global a los 5 años de los pacientes con somatostatinoma es del $40 \%$ al $60 \%{ }^{6}$ La sobrevida a los 5 años de los somatostatinomas con metástasis hepáticas es del $40 \%$, sin embargo, en los tumores sin metástasis hepática o ganglionar es del $100 \%{ }^{6}$.

5. Krejs GJ, Orci L, Conlon JM, Ravazzola M, Davis GR, Raskin Pet al. Somatostatinoma syndrome. Biochemical, morphologic and clinical features. N Engl J Med1979;301: 285-92

6. House MG, Yeo CJ, Schulick RD.Periampullary pancreatic somatostatinoma. Ann Surg Oncol 2002; 9:869-74.

7. Mullen JT, Wang H, Yao JC, Lee JH, Perrier ND, Pisters PWet al. Carcinoid tumors of the duodenum.Surgery 2005;138:971-7.

8. Kim JA, Choi W, Kim CN, Moon YS, Chang SH, Lee HR. Duodenalsomatostatinoma: a case report and review. Korean J of Internal Med 2011;1:26.

9. Tanaka S, Yamasaki S, Matsushita H, Ozawa Y, Kurosaki A, Takeuchi Ket al. Duodenal somatostatinoma:a case report and review of 31 cases with special reference to the relationship between tumor size and metastasis. PatholInt 2000;50:146-52. 